\title{
619 PHARMACOLOGIC MODULATION OF TUMOR GLYCOLYSIS TO IMPROVE RESPONSES TO IMMUNE CHECKPOINT BLOCKADE THERAPY
}

${ }^{1}$ Svena Verma*, ${ }^{1}$ Rachana Maniyar, ${ }^{1}$ Myat Ko, ${ }^{1}$ Sadna Budhu, ${ }^{2}$ Inna Serganova, ${ }^{2}$ Roberta Zappasodi, ${ }^{1}$ Ronald Blasberg, ${ }^{1}$ Taha Merghoub, ${ }^{1}$ Jedd Wolchok, ${ }^{1}$ Levi Mangarin. ${ }^{1}$ Memorial Sloan Kettering, New York, NY, USA; ${ }^{2}$ Weill Cornell Medicine, New York, NY USA

Background Immune checkpoint blockade (ICB) has revolutionized the treatment of many cancer types; however, many patients do not benefit from these therapies due to inherent or acquired resistance. Preferential engagement in glycolysis is a hallmark of cancer cells and contributes to the progression and metastasis of many tumor types, including melanoma and triple-negative breast cancer (TNBC). Tumor reliance on glycolysis is emerging as a mechanism of resistance to immunotherapy, due in part to lactate-mediated acidification and competition for glucose in the tumor microenvironment. We recently found that knocking down the glycolytic enzyme lactate dehydrogenase A (LDHA) in 4T1 TNBC results in improved and long-lasting anti-tumor responses to CTLA-4 blockade in mice. These LDHA-defective tumors consume less glucose than wild-type tumors, leaving more glucose available in the TME for effector $\mathrm{T}$ cells to exert their tumoricidal function directly and via lineage destabilization of regulatory $\mathrm{T}$ cells. However, it remains to be determined whether systemic pharmacologic targeting of $\mathrm{LDH}$ can improve the efficacy of immunotherapy.

Methods Lactate production and glucose consumption was quantified by YSI enzymatic analysis of metabolites. LDHA was detected by immunoblot in tumor and $\mathrm{T}$ cells. A clonogenic killing assay was used to assess T-cell killing. B16-F10bearing mice were treated daily with $\mathrm{LDH}$ inhibitor GNE-140 and/or biweekly with anti-CTLA-4.

Results Since activated T cells rely on glycolysis, we first determined that glycolytic cancers overexpress LDH compared to immune cells by analyzing single-cell transcripts from patient melanoma biopsies. LDHA gene expression is significantly higher in malignant cells than infiltrating CD8 $+\mathrm{T}$ cells, and we replicated these findings at the protein level in whole cell lysate from B16-F10 melanoma and 4T1 TNBC tumor cells vs. activated tumor-antigen specific $\mathrm{T}$ cells in vitro. We showed that the LDH inhibitor GNE-140 reduces tumor lactate production and glucose consumption without inhibiting anti-tumor T-cell killing in vitro. Daily treatment with GNE140 results in reduced growth in immunocompetent but not immune deficient mice. Additionally, our preliminary findings indicate that targeting LDHA in combination with CTLA-4 blockade is more effective in slowing B16-F10 growth compared with CTLA-4 blockade alone.

Conclusions These results suggest that targeting $\mathrm{LDH}$ with GNE-140 is a safe, efficacious method for relieving tumor glycolysis-mediated immunosuppression within the TME, without adversely affecting immune cell function within the tumor. Our long-term goal is to determine optimal combinations of metabolic inhibitors with ICB to alleviate the metabolic competition between tumor and infiltrating immune cells and better potentiate anti-tumor immune responses.

http://dx.doi.org/10.1136/jitc-2021-SITC2021.619 